\title{
Editorial
}

Journal of Innate

Immunity

\section{Participation of Host Determinants in the Pathogenesis of Pneumonia}

\author{
Alice Prince \\ Departments of Pediatrics and Pharmacology, Columbia University Medical Center, New York, NY, USA
}

In this respiratory theme issue, we address some of the newer concepts and strategies to elucidate the complexity of host-pathogen interactions in the lung. Much of the microbial pathogenesis literature has focused upon the microbe, delineating specific virulence factors and their targets. This approach has been tremendously fruitful. Numerous bacterial and viral virulence factors have been defined and verified through murine models of pneumonia using both microbial and murine mutants. The potent consequences of inflammasome activation, induced by numerous bacterial toxins, flagellins, and components of the type 3 secretion system, have been characterized, and their participation in host defense has been determined in these models. How various microbial PAMPs activate NF- $\mathrm{KB}, \mathrm{TNF}$, and MAPK signaling pathways are increasingly well understood as these are important in recruiting and activating immune cells critical for an appropriate immune response. Somewhat less clear is how the host appropriately responds to the inflammation that is induced, and how coincident tissue damage is prevented and/or repaired.

It is well appreciated that different hosts respond very differently to the exact same pathogen. However, the specific factors that are responsible for this diversity are poorly understood. As reinforced by the articles in this

\section{KARGER}

() 2017 S. Karger AG, Basel

E-Mail karger@karger.com

www.karger.com/jin issue, the extent of host innate immune response activation is critical in determining outcome. There are multiple redundant proinflammatory mechanisms activated in stromal and immune cells through NF- $\kappa \mathrm{B}, \mathrm{MAPK}$, TNF, and inflammasome signaling. The regulation of these pathways, at any of the multiple stages in protein expression, could provide potential targets for therapy. Conserved inflammatory, cell death, and even proliferative responses are evoked by infection and have been successfully targeted in oncology and rheumatology. However, host variability and the tremendous diversity in gene expression and regulation even within the same microbial species present major challenges.

The transcriptome response to influenza has been well studied as the identical virus can be given to DBA2 mice that are susceptible to infection with $100 \%$ mortality, whereas the C57Bl6 mice are entirely resistant. As summarized in his review, Kobzik [1] utilized multiple available GWAS data sets to try to identify host susceptibility and resistance loci from well-controlled murine experiments that would be relevant to the epidemiology and outcomes associated with human influenza. From his analysis, reviewing both the primary data as well as performing a meta-analysis, it appears that an excessive proinflammatory response is associated with a poor out- 
come, not the inability to mount an immune response. Other major pathways associated with outcome included cell death pathways as well as proliferation pathways. Additional studies using the mouse model of influenza further documented that suppression of inflammation in the setting of acute influenza significantly improved outcome and documented the importance of dysregulated inflammation as a major cause of pathology and mortality in influenza. Such GWAS have many limitations, especially their failure to assess posttranscriptional mechanisms to regulate immune responses. However, as well illustrated in several other reviews, the central themes of excessive inflammation, impaired regulation of cell death pathways, and proliferative responses are consistently illustrated in the pathological responses to diverse types of infection.

The molecular mechanisms to regulate expression of the pulmonary epithelial genes involved in inflammation and, importantly, in repair processes are reviewed by Cohen [2]. His review focuses upon the importance of microRNA (mIR) expression in pulmonary infection. There is increasing appreciation of mIRs and c-Myc as a central regulator of multiple mIRs that control epithelial proliferative responses. Cellular proliferation is activated as part of the repair process following severe infection, a process targeted by several mIRs. This review also draws upon the influenza literature and the transcriptome studies, to highlight data documenting the participation of mIR-221 in epithelial regeneration after influenza. The many roles of $\mathrm{mIRs}$ in proliferative regulation are well appreciated in oncology, and similar processes are very likely to be relevant to mount the appropriate response to influenza.

The theme of excessive host inflammation and associated pathology is also the topic reviewed by Lin and Kazmierczak [3]. These authors examine the mechanisms through which Pseudomonas aeruginosa cause lung damage by activation of inflammasome- and NF- $\kappa \mathrm{B}$-mediated responses. The pathological changes associated with $P$. aeruginosa pneumonia are not entirely dissimilar from the features of fatal influenza, as the inflammatory response to infection is not appropriately controlled by the host. As suggested by the authors, improved outcomes of pulmonary infection due to $\mathrm{P}$. aeruginosa have been linked to the inhibition of inflammasome-mediated signaling through antagonists of IL-1R, e.g., anakinra, a drug now in use for rheumatologic diseases.

Innate immune mechanisms to control excessive inflammation in the lung are also presented. Specifically the role of necroptosis, a RipK1/Ripk3/Mlkl-mediated mech- anism of cell death, is reviewed in this issue [4]. Cell death functions as an immunoregulatory mechanism by removing infected cells that can participate in proinflammatory signaling. Just as cell death pathways featured prominently in the transcriptomic studies of influenza outcomes, they have also been shown to be important in bacterial pneumonia. Multiple bacterial toxins activate necroptosis, as reviewed by Ahn and Prince [4]. In the lung, Staphylococcus aureus-induced necroptosis eliminates an important population of regulatory macrophages, thus contributing to excessive inflammation. However, for other pathogens, cell death through toxininduced necroptosis improves bacterial clearance. Hostadapted pathogens, such as the ST258 Klebsiella pneumoniae, may avoid activating the necroptosis pathway entirely.

The commonality of the host responses to the diverse infections reviewed suggests that several components of the host response, particularly the IL-1 pathway, perhaps necroptosis, or targeting specific mIRs, may prove to be therapeutic targets useful in managing lung pathology due to infection. The discussion of innate immune signaling involved in sensing and responding to the invasive opportunist Aspergillus fumigatus, reviewed by Garth and Steele [5], clearly illustrates the complexities of the hostpathogen interaction. Although chiefly an infection of neutrophil-deficient immunocompromised hosts, these authors also reinforce the concept that the appropriate regulation of immune signaling in response to A. fumigatus infection is critical, and involves numerous discrete pattern recognition receptors on a variety of stromal and immune cells.

Overall, the intent of these articles is to highlight the complexity of innate immune responses to pulmonary infection and new approaches to define the genetic basis for these responses, and how they can be regulated. These reviews illustrate that despite the complexity of host responses and pathogen adaptation, there are shared pathways that could provide targets to correct dysregulated immune signaling. It is important to recognize that many of these pathways, such as the MAPKs, IL-1R signaling, proliferative cascades, and several other pathways, have already been targeted in oncology and/or rheumatology, and are the basis for drugs in clinical use. Thus, identifying the causes of the dysregulated immune responses to distinct infections may prompt the development of specific therapies. 


\section{References}

1 Kobzik L: Searching for a lifeline: transcriptome profiling studies of influenza susceptibility and resistance. J Innate Immun 2017; 9:232-242.

2 Cohen TS: Role of microRNA in the lung's innate immune response. J Innate Immun 2017;9:243-249.
3 Lin CK, Kazmierczak BI: Inflammation: a double-edged sword in the response to Pseudomonas aeruginosa infection. J Innate Immun 2017;9:250-261.

4 Ahn D, Prince A: Participation of necroptosis in the host response to acute bacterial pneumonia. J Innate Immun 2017;9:262-270.

5 Garth JM, Steele C: Innate lung defense during invasive aspergillosis: new mechanisms. J Innate Immun 2017;9:271-280. 